\title{
Elderly Bleeding Risk of Direct Oral Anticoagulants in Nonvalvular Atrial Fibrillation: A Systematic Review and Meta-Analysis of Cohort Studies
}

\author{
Jessika Lobraico-Fernandez ${ }^{1} \cdot$ Salma Baksh $^{2} \cdot$ Eric Nemec $^{1}$ (1)
}

Published online: 24 May 2019

(c) The Author(s) 2019

\begin{abstract}
Introduction The 2014 American Heart Association (AHA)/American College of Cardiology (ACC)/Heart Rhythm Society (HRS) guidelines recommend anticoagulation to reduce clot formation and the risk of thromboembolic events in patients with atrial fibrillation but does not specify guidelines for the elderly population. Direct oral anticoagulants (DOACs) are newer US FDA-approved alternatives to warfarin and include dabigatran, rivaroxaban, apixaban and edoxaban. The efficacy of DOACs is heavily researched, but few studies have evaluated their bleeding risk.

Objectives This systematic review and meta-analysis investigates which DOAC has the lowest bleeding risk in elderly patients with nonvalvular atrial fibrillation (NVAF).

Methods CINAHL and MEDLINE databases were searched using specific keywords, and 244 results were identified and screened. Inclusion criteria required a major bleeding event requiring hospitalization as an outcome and excluded patients with severe renal failure. Articles that met inclusion criteria were assessed for risk of bias using the Cochrane Tool to Assess Risk of Bias in Cohort Studies. Review Manager (version 5) was used to perform the random-effects model meta-analysis. Hazard ratios (HRs) and 95\% confidence intervals (CIs) were calculated. $P<0.05$ was considered statistically significant.

Results Six articles met inclusion criteria and encompassed 446,042 patients in total. Apixaban and dabigatran had statistically significant risk reductions compared with warfarin, whereas rivaroxaban did not (HR 0.60 [95\% CI 0.52-0.69], $p<0.00001$; HR 0.79 [95\% CI 0.70-0.90], $p=0.0005$; HR 1.03 [95\% CI 0.86-1.22], $p=0.77$, respectively.) Data regarding edoxaban were limited and thus not included in the analysis.

Conclusion Apixaban and dabigatran have a significantly decreased major bleeding risk ( 40 and $21 \%$, respectively) compared with warfarin. There was no statistical difference in bleeding risk between rivaroxaban and warfarin. Head-to-head prospective randomized controlled trials are required to assess the true bleeding risk of each DOAC.
\end{abstract}

\section{Key Points}

In patients aged $>65$ years, apixaban and dabigatran have a significantly decreased major bleeding risk, 40 and $21 \%$, respectively, compared with warfarin.

In this population, there was no significant difference in bleeding risk between rivaroxaban and warfarin.

Providers should consider the relative bleeding risk when prescribing anticoagulants to older patients.

Eric Nemec

nemece@ sacredheart.edu

1 Sacred Heart University Physician Assistant Studies, Fairfield, CT, USA

2 Columbia University Medical Center, New York Presbyterian Hospital, New York, NY, USA

\section{Introduction}

Atrial fibrillation (AF) affects an estimated 2.7-6.1 million people in the USA, and the incidence is expected to rise as the population ages and life expectancies increase [1]. The prevalence of $\mathrm{AF}$ is higher in older and elderly populations, at about $9 \%$ of people aged $>65$ years and $2 \%$ of those aged $<65$ years [7]. Current 2014 American Heart Association (AHA)/ American College of Cardiology (ACC)/Heart Rhythm Society (HRS) guidelines recommend that clinicians control their patients' heart rhythm or rate and prescribe an anticoagulant to reduce clot formation and risk of a thromboembolic event [1]. Further, the AHA reports that the risk of stroke in a patient with $\mathrm{AF}$ is four to five times higher than in an individual without it, so it is imperative that patients in the general population are anticoagulated [7]. Anticoagulation of the elderly population is less extensively studied, and the risks and safety of direct oral anticoagulants (DOACs) need further evaluation. 
Anticoagulant options for nonvalvular atrial fibrillation (NVAF) include warfarin and the newer DOACs, dabigatran, rivaroxaban, apixaban, and edoxaban [1,2]. Warfarin is a vitamin $\mathrm{K}$ inhibitor that has been shown to effectively prevent stroke and reduce all-cause mortality in patients with NVAF [8]. However, it also requires regular monitoring of the international normalized ratio (INR) and dose adjustment to ensure the patient is within the narrow therapeutic range of 2.0-3.0, in the absence of a mechanical valve. Because of this clinical burden and associated potential bleeding risk, the nonvitamin $\mathrm{K}$ inhibitors, also known as the aforementioned DOACs, were developed. ROCKET, RE-LY, ARISTOTLE, and ENGAGE AF-TIMI were landmark trials that resulted in US FDA approval of DOACs and provided pivotal data of the noninferiority and similar efficacy of rivaroxaban, dabigatran, apixaban, and edoxaban, respectively, as compared with warfarin [2-5].

Given the results of these large and well-known studies, the DOACs are now commonly prescribed to patients with NVAF because of their efficacy and safety as well as their lack of need for INR monitoring and dose adjustment. Of note, the current AHA/ACC/HRS guidelines for AF management were released in 2014 and do not include edoxaban, which received FDA approval in 2015. This relatively recent approval means very little literature exists that compares edoxaban with the other DOACs and warfarin.

Most DOAC studies have focused on the rate of thromboembolism and stroke prevention compared with warfarin, but no head-to-head clinical trials have assessed the bleeding risk of each DOAC to determine best practice for prescribing to patients [9]. Additionally, no recommendations are currently available regarding which DOAC to prescribe to patients, and the decision is often made according to provider preference, in conjunction with patient input and affordability.

While this a practical approach to prescribing, different DOACs may be superior in different patients with various comorbidities and other contributing factors (such as age, lifestyle, compliance concerns, etc.). Of note, the prevalence of AF is $8-34 \%$ in patients with chronic kidney disease undergoing hemodialysis, a disease that generally progresses with advanced age [10]. While NVAF is commonly encountered in severe renal failure, posing an increased risk of systemic thromboembolism, scant literature is available regarding DOAC recommendations. DOAC use for the prevention of thromboembolism or stroke in patients with NVAF and severe renal failure also requires dosing adjustment or may be contraindicated, depending on the DOAC and individual measurements of renal function. The use of DOACs in these patients also poses an increased bleeding risk; thus, drug choice, dosing, and monitoring should be carefully considered before prescribing.
With the large majority of patients with NVAF being aged $>65$ years, anticoagulation is common, but this population is also at increased risk of other associated complications, including major bleeding [1, 11]. In addition to incidental intracranial or gastrointestinal bleeding in this population, the World Health Organization (WHO) reports an annual fall risk of $30 \%$ in community-dwelling people aged $>65$ years [12]. This risk, combined with anticoagulation in NVAF, imposes a risk of traumatic bleeding in addition to idiopathic bleeds in this population [13].

The ACC reported that a major hurdle to DOAC popularity is their safety regarding bleeding risk and concern about a lack of reversal agents [14]. However, this should also be even less of a concern now, because, in addition to a reversal protocol for a warfarin-related bleed, all three major DOACs now have FDA-approved reversal agents $[15,16]$. DOACs may be better suited anticoagulants for older patients with NVAF given the presence of reversal agents, simplicity in dosing, a lack of need for INR monitoring, as well as a potentially lower bleeding risk compared with warfarin. This review seeks to determine which DOAC has the lowest bleeding risk in patients with NVAF aged $>65$ years.

\section{Methodology}

\subsection{Data Sources and Search Strategy}

Both CINAHL Complete and MEDLINE with Full Text were searched concomitantly using EBSCO Information Services on 12 July 2018 and 13 July 2018 using the following key terms: "dabigatran OR apixaban OR rivaroxaban OR warfarin OR direct oral anticoagulant OR DOAC" AND "non-valvular atrial fibrillation OR atrial fibrillation" AND "bleed OR bleeding OR hemorrhage OR bleed risk OR bleeding risk OR hemorrhage risk". All duplicate articles were removed, and the following limits were applied: timeframe (2008-present), language (English), age of subjects ( $>65$ years), and peer-reviewed articles. A gray literature search was performed using the National Institute for Health and Care Excellence (NICE) database and the PROSPERO Clinical Trials Database using the same key terms. No additional studies were identified during the gray literature search. Search results were exported from EBSCO into Covidence systematic review manager for screening and selection by two independent reviewers. Discrepancies were resolved via discussion.

\subsection{Eligibility Criteria}

Inclusion criteria were defined as follows: NVAF; includes apixaban, dabigatran and rivaroxaban; may or 
may not include warfarin; age $>65$ years; retrospective propensity-matched cohort studies; and primary outcome of major bleed or bleed risk. Exclusion criteria were defined as valvular or other causes of AF, wrong comparator (does not include apixaban, dabigatran, or rivaroxaban), studies focused on those aged $<65$ years, review articles, and clinical trials. Clinical trials were excluded because a lack of method uniformity meant data compilation and making conclusions about outcomes were more difficult and because head-to-head DOAC clinical trials were scarce. Studies that used the same databases with overlapping patient data were evaluated to determine which were most inclusive of patients and had the largest timeframe.

The primary endpoint was bleed rate or risk with each DOAC compared with each other or with warfarin. A PRISMA (Preferred Reporting Items for Systematic Reviews and Meta-Analyses) statement flow diagram was used to report the review exclusion process and article selection.

\subsection{Risk of Bias Evaluation}

Risk of bias was evaluated in all included studies using the Cochrane Tool to Assess Risk of Bias in Cohort Studies. This tool assesses eight aspects, including subject selection, assessment of exposure, confidence in baseline, confounding variables, prognostic factors, outcome assessment, followup, and co-intervention. The eight questions are assessed on a four-point scale: low risk, moderately low risk, moderately high risk, and high risk of bias. In this review, we included only studies assessed as having a low risk or moderately low risk of bias for all eight questions.

\subsection{Meta-Analysis}

A meta-analysis was conducted using the five included studies that evaluated a major bleed. Review Manager (RevMan) software, version 5 (Copenhagen: the Nordic Cochrane Centre, The Cochrane Collaboration, 2008) was used to perform the meta-analysis following the recommendations of the Cochrane Collaboration. Hazard ratios (HRs) and 95\% confidence intervals (CIs) were calculated for dichotomous outcomes. The $\chi^{2}$ test and $I^{2}$ statistics were calculated to determine heterogeneity, which was considered significant when the $p$ value from $\chi^{2}$ was $<0.10$ or when the $I^{2}$ statistic was $>50 \%$. A random-effects model was selected, and a funnel plot was created to evaluate publication bias. Statistical significance was accepted when $p<0.05$.

\section{Results}

\subsection{Study Selection}

The specified search strategy identified 256 articles. Following import into Covidence, 12 duplicates were removed. In total, 244 studies were screened and 160 were excluded because they were irrelevant. Full-text assessment for eligibility was performed on 84 studies, and 78 were excluded for reasons indicated in the PRISMA diagram (Fig. 1). Six studies were identified as propensity score-matched cohorts and were assessed for risk of bias (see Sect. 2.3). One study was identified as eligible but excluded because of a database overlap with another study, and the chosen study included a larger more expansive timeframe [17, 18]. All six studies had the lowest risk of bias for each of the eight questions and were included in this review.

\subsection{Study Characteristics}

All studies included in this review evaluated patients with NVAF and their bleeding risk while receiving one of the following anticoagulants: apixaban, dabigatran, rivaroxaban, or warfarin. Propensity score-matched cohorts were utilized in all studies to reduce the impact of confounders on the primary outcome. Four studies compared a DOAC with warfarin, one study compared each DOAC with other DOACs, and the remaining study compared each DOAC with both warfarin and each DOAC [6, 18-22]. The follow-up period for studies ranged from 50 days to 2.5 years, with day 1

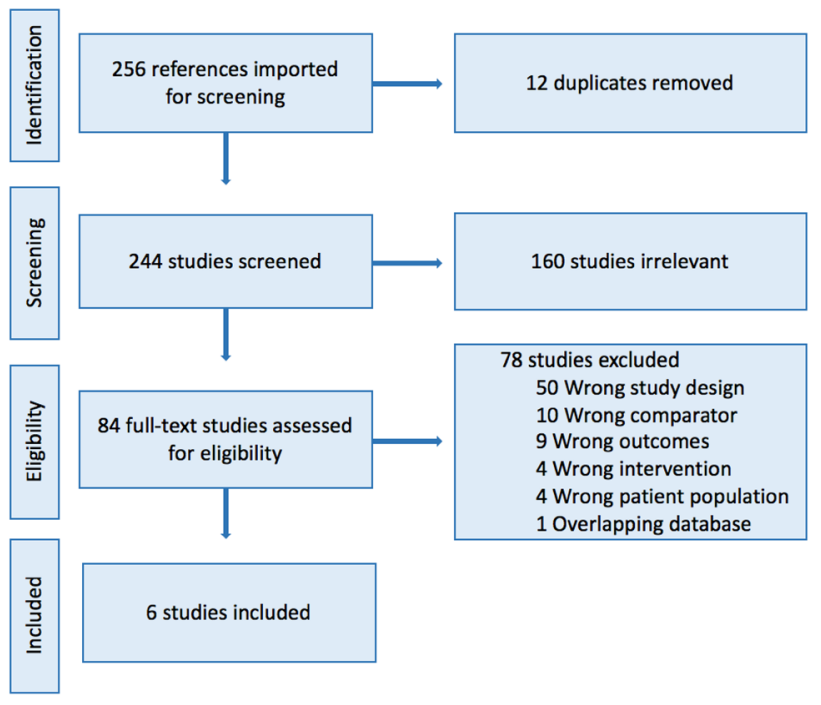

Fig. 1 PRISMA Flow Diagram 
being the index date of prescription filled. The primary outcome was a major or gastrointestinal bleed. The definition of a major bleed included bleed requiring hospitalization through to bleeding at major sites, including but not limited to intracranial, gastrointestinal, liver, splenic, and ocular. Patients were identified in databases using International Classification of Diseases, Ninth Edition (ICD-9) codes or variations of this coding system in other countries as well as billing and pharmacologic records. Sample sizes ranged from 38,622 to 186,132 patients. The origin of patient populations included the USA [6, 19, 20, 22], Japan [21], and Denmark [18]. Table 1 displays the specific characteristics of each study.

\subsection{Risk of Bias}

Based upon the Cochrane Tool to Assess Risk of Bias in Cohort Studies, the risk of bias was low for all six studies: they all used large, reliable data sources with access to insurance claims and hospital coding. All studies also utilized propensity score matching to reduce confounders and account for covariates that may impact the risk of bleed outcome.

\subsection{Participant Characteristics}

This review includes 446,042 patients diagnosed with NVAF and receiving anticoagulation for the first time or who had not been anticoagulated for at least 2 years before the start of each study [6, 18-22]. Most included studies reported patient characteristics within each propensityscore matched cohort rather than as a summative depiction, so this review provides the ranges of mean age and percentage of males included in each study, as shown in Table 2. The mean age of studies reported in this review ranges from 66.3 to 78.4 years, and the percentage of males ranges between 47.4 and $66.1 \%$. Comorbidities were not reported in this review because those that would impact bleeding risk, such as renal disease, were excluded from included studies. Further, patients were propensity score matched based on similar demographic and clinical characteristics to best minimize confounding variables.

\subsection{Major Bleed Risk Outcomes}

All studies reported bleeding risk using HRs. Beyond major bleeds, two studies reported data on gastrointestinal bleed risk and one reported data on both gastrointestinal bleed and

Table 1 Study characteristics

\begin{tabular}{|c|c|c|c|c|c|}
\hline Study & Patients $(N)$ & Drugs evaluated & Comparison group & $\begin{array}{l}\text { Median length of } \\
\text { follow-up, days }\end{array}$ & Data resource \\
\hline Abraham et al. [6] & 43,303 & API, DAB, RIV & $\begin{array}{l}\text { API vs. DAB, } \\
\text { API vs. RIV } \\
\text { DAB vs. RIV }\end{array}$ & $\begin{array}{l}\text { API 89; DAB } 120 \\
\text { API 89; RIV } 106 \\
\text { DAB 120; RIV } 113\end{array}$ & $\begin{array}{l}\text { OptumLabs Data Warehouse (contains commer- } \\
\text { cial and Medicare Advantage data) }\end{array}$ \\
\hline Amin et al. [19] & 186,132 & API, DAB, RIV & $\begin{array}{l}\text { API vs. WAR } \\
\text { DAB vs. WAR } \\
\text { RIV vs. WAR }\end{array}$ & $\begin{array}{l}\text { API 115; WAR } 122 \\
\text { DAB 113; WAR } 126 \\
\text { RIV 130; WAR } 120\end{array}$ & US Center of Medicare and Medicaid Services \\
\hline Kohsaka et al. [21] & 38,662 & API, DAB, RIV & $\begin{array}{l}\text { API vs. WAR } \\
\text { DAB vs. WAR } \\
\text { RIV vs. WAR }\end{array}$ & $\begin{array}{l}\text { API 82; WAR } 50 \\
\text { DAB 154; WAR } 148 \\
\text { RIV 99; WAR } 67\end{array}$ & $\begin{array}{l}\text { Medical Data Vision Company Japanese Data- } \\
\text { base }\end{array}$ \\
\hline Li et al. [22] & 76,940 & API & API vs. WAR & API 119; WAR 122 & $\begin{array}{l}\text { US Truven MarketScan, PharMetrics, Optum } \\
\text { and Humana Databases }\end{array}$ \\
\hline Lip et al. [20] & 45,361 & API, DAB, RIV & $\begin{array}{l}\text { API vs. WAR } \\
\text { DAB vs. WAR } \\
\text { RIV vs. WAR } \\
\text { API vs. DAB } \\
\text { API vs. RIV } \\
\text { DAB vs. RIV }\end{array}$ & $\begin{array}{l}\text { API 96; WAR } 100 \\
\text { DAB 100; WAR } 97 \\
\text { RIV 113; WAR } 100 \\
\text { API 93; DAB } 103 \\
\text { API 95; RIV } 116 \\
\text { DAB 100; RIV } 111\end{array}$ & $\begin{array}{l}\text { US Truven MarketScan Commercial Claims and } \\
\text { Encounter and Medicare Supplemental and } \\
\text { Coordination of Benefits Databases }\end{array}$ \\
\hline Nielsen et al. [18] & 55,644 & API, DAB, RIV & $\begin{array}{l}\text { API vs. WAR } \\
\text { DAB vs. WAR } \\
\text { RIV vs. WAR }\end{array}$ & 2.5 years for all & $\begin{array}{l}\text { Danish National Prescription Registry, Danish } \\
\text { National Patient Register, Danish Civil Regis- } \\
\text { tration System }\end{array}$ \\
\hline
\end{tabular}

Major bleed includes bleeding at key sites, including but not limited to intracranial, gastrointestinal, liver, splenic and ocular hemorrhage

$A P I$ apixaban, DAB dabigatran, RIV rivaroxaban, WAR warfarin

${ }^{a}$ Unless otherwise specified 
Table 2 Participant characteristics

\begin{tabular}{lll}
\hline Study & Age, mean \pm SD & Sex $(\%$ male $)$ \\
\hline Abraham et al. [6] & $69.2 \pm 11.6$ to $72.3 \pm 11.1^{\mathrm{a}}$ & $53.9-59.7^{\mathrm{b}}$ \\
Amin et al. [19] & $77.1 \pm 7.3$ to $78.4 \pm 7.4^{\mathrm{a}}$ & $47.4-51.0^{\mathrm{b}}$ \\
Kohsaka et al. [21] & $73.1 \pm 9.9$ to $77.7 \pm 10.0^{\mathrm{a}}$ & $58.9-66.1^{\mathrm{b}}$ \\
Li et al. [22] & $70.9 \pm 11.9-12.0^{\mathrm{a}}$ & $59.7-59.8^{\mathrm{b}}$ \\
Lip et al. [20] & $66.3 \pm 12.3-70.1 \pm 12.0^{\mathrm{a}}$ & $60.9-65.0^{\mathrm{b}}$ \\
Nielsen et al. [18] & $73.9 \pm 12.7$ & $55.1 \%$ \\
\hline
\end{tabular}

$S D$ standard deviation

${ }^{a}$ Ranges reported due to differences between propensity scorematched cohorts

${ }^{\mathrm{b}}$ Reported as median

intracranial bleed risk [6, 19]. When comparing each DOAC with warfarin, warfarin was considered the reference standard. Only one study reported data on major bleed risk when comparing DOACs with each other and with warfarin [20].

\subsubsection{Apixaban}

Apixaban appears to have the lowest major bleeding risk, with four studies reporting between $21 \%$ (HR 0.79 [95\% CI 0.65-0.96]) and 49\% (HR 0.51 [95\% CI 0.44-0.58]) compared with warfarin, shown in Table 3 [18-21]. Further, all four studies that evaluated major bleed risk reported a lower risk with apixaban than with dabigatran and rivaroxaban compared with warfarin.

Following meta-analysis, apixaban had a statistically significant $40 \%$ reduction in major bleeding risk (HR 0.60 [95\% CI 0.52-0.69] $p<0.00001)$, as shown in Table 4. The dataset is also considered heterogenous, with a $\chi^{2}$ of 12.55 and $I^{2}$ of $68 \%$. Further, the heterogeneity and low publication bias of the studies included in the meta-analysis of apixaban is evidenced by the funnel plot in Fig. 2 .

\subsubsection{Dabigatran}

Four of the six included studies also suggested that dabigatran has a statistically significant lower risk of major bleed than warfarin, with the risk reduction ranging from $21 \%$ (HR 0.79 [95\% CI 0.69-0.91]) to 38\% (HR 0.62 [95\% CI 0.43-0.90]), shown in Table 5. While these rates are similar to those reported in this review for apixaban, it is important to note that the majority of included studies reported statistically significant and greater risk reduction with apixaban than with warfarin. Further, when bleeding risk was assessed between apixaban and dabigatran, dabigatran had a $41 \%$ greater risk than apixaban for a major bleed (HR 1.41 [95\% CI 0.93-2.14]) but did not reach statistical significance [20].

Table 3 Apixaban bleeding risk

\begin{tabular}{llll}
\hline Apixaban vs. & Major bleed & Gastrointestinal bleed & Intracranial bleed \\
\hline Warfarin & $0.51^{*}(0.44-0.58)[19]$ & $0.63^{*}(0.52-0.76)[19]$ & $0.38^{*}(0.25-0.56)[19]$ \\
& $0.59^{*}(0.42-0.82)[21]$ & $0.62^{*}(0.55-0.71)[22]$ & $0.64 *(0.50-0.80)[22]$ \\
& $0.60^{*}(0.54-0.65)[22]$ & & \\
& $0.79 *(0.65-0.96)[19]$ & & NA \\
Dabigatran & NA & $0.39 *(0.27-0.58)[6]$ & NA \\
Rivaroxaban & NA & $0.33^{*}(0.22-0.49)[6]$ & \\
\hline
\end{tabular}

Data are presented as hazard ratio (95\% confidence interval)

$N A$ data not available

$* p<0.05$

Table 4 Apixaban bleeding risk forest plot

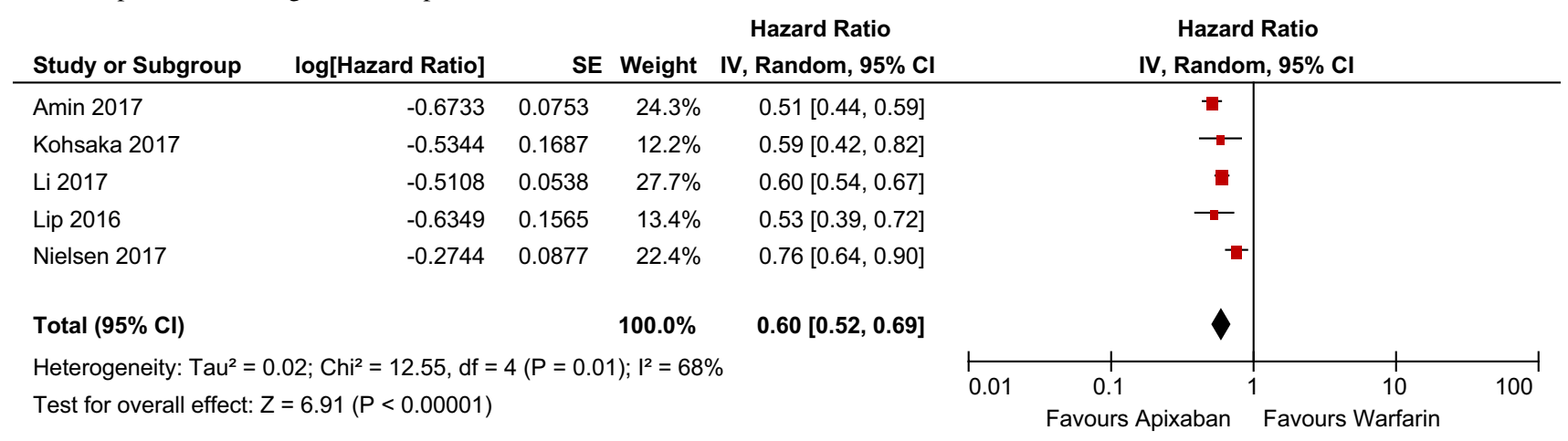

$C I$ confidence interval, $S E$ standard error 


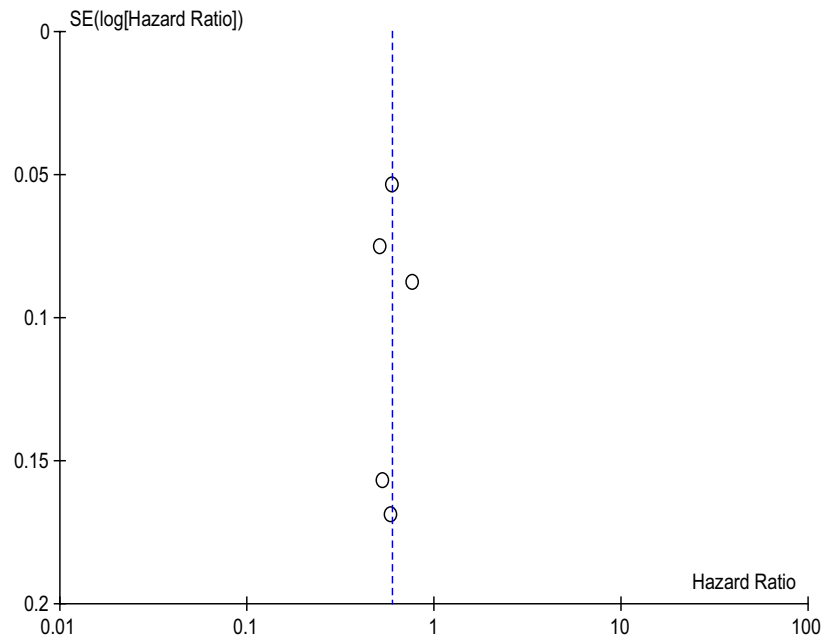

Fig. 2 Apixaban Funnel Plot

Meta-analysis of the included studies determined that dabigatran has a $21 \%$ reduction in major bleeding risk compared with warfarin (HR 0.79 [95\% CI 0.70-0.90], $p=0.0005)$, shown in Table 6 . The included studies are also considered moderately heterogeneous, with a $\chi^{2}$ of 5.08 and an $I^{2}$ of $41 \%$. Further, the funnel plot for included dabigatran studies exhibits low publication bias, as evidenced by Fig. 3 .

\subsubsection{Rivaroxaban}

The major bleeding risk with rivaroxaban was comparable to that with warfarin in three studies, shown in Table $7[18,20]$. In addition, one study showed a statistically significant risk reduction of $31 \%$, and another showed a $17 \%$ significantly increased risk of major bleed compared with warfarin (HR 0.69 [95\% CI 0.51-0.93]), HR 1.17 [95\% CI 1.10-1.26], respectively) $[19,21]$. When compared with apixaban, rivaroxaban incurred an $82 \%$ increased risk of bleeding (HR 1.82 [95\% CI 1.36-2.43]) [20]. In comparison with dabigatran, major bleeding risk with rivaroxaban was not statistically significantly different [20].

Meta-analysis indicated that the bleeding risk with rivaroxaban was comparable to that with warfarin (HR $1.03[95 \%$ CI 0.86-1.22], $p=0.77$ ), shown in Table 8. These results are drawn from a heterogeneous dataset with a $\chi^{2}$ of 14.62 and $I^{2}$ of $79 \%$. Further, the funnel plot displayed in Fig. 4 provides evidence for a low publication bias and heterogeneity of included studies.

\subsection{Gastrointestinal Bleeding Risk}

As displayed in Tables 3, 5, and 7, the risk of a gastrointestinal bleed while taking apixaban was lower by $37 \%$ (HR 0.63 [95\% CI 0.52-0.76]) and 38\% (HR 0.62 [95\% CI $0.55-0.71]$ ), both statistically significant compared with
Table 5 Dabigatran bleeding risk

\begin{tabular}{llll}
\hline Dabigatran vs. & Major bleed & Gastrointestinal bleed & Intracranial bleed \\
\hline Warfarin & $0.79 *(0.69-0.91)[19]$ & $1.02(0.85-1.23)[19]$ & $0.54 *(0.35-0.82)[19]$ \\
& $0.62 *(0.43-0.90)[21]$ & & \\
& $0.69 *(0.50-0.96)[20]$ & & \\
& $0.89(0.78-1.02)[18]$ & & NA \\
Apixaban & $1.41(0.93-2.14)[20]$ & NA & NA \\
Rivaroxaban & $1.05(0.74-1.49)[20]$ & NA & \\
\hline
\end{tabular}

Data are presented as hazard ratio ( $95 \%$ confidence interval)

$N A$ data not available

$* p<0.05$

Table 6 Dabigatran bleeding risk forest plot

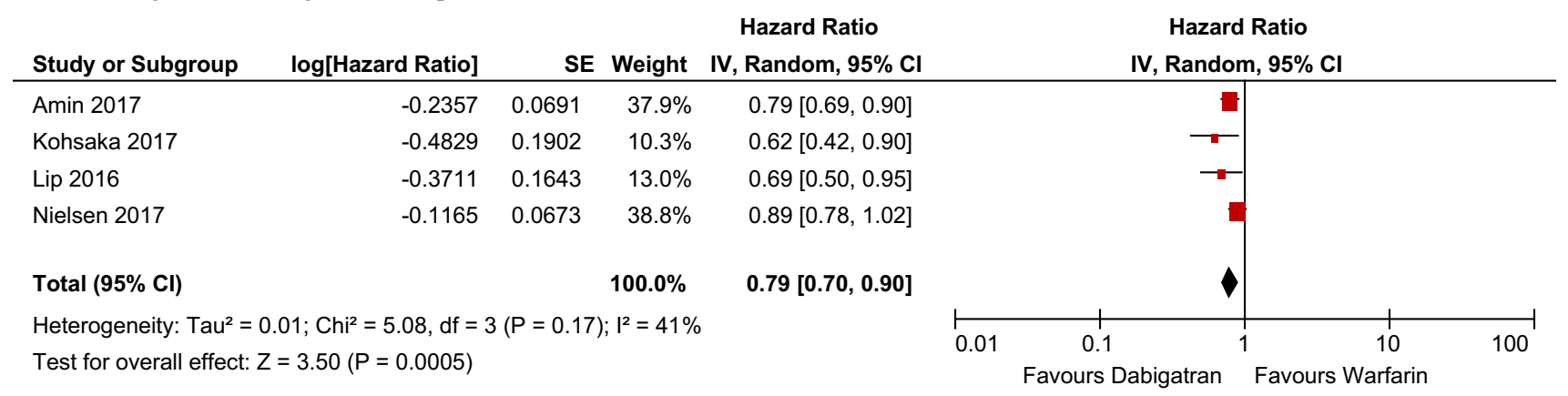

$C I$ confidence interval, $S E$ standard error 


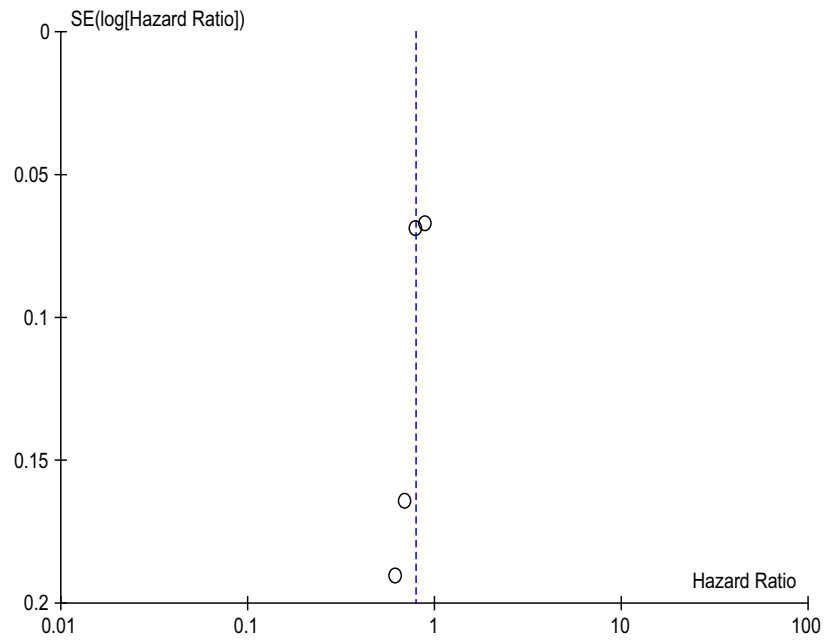

Fig. 3 Dabigatran Funnel Plot

warfarin $[19,22]$. The risk with apixaban was $61 \%$ (HR 0.39 [95\% CI $0.27-0.58]$ ]) lower than with dabigatran and $67 \%$ (HR 0.33 [95\% CI 0.22-0.49]) lower than with rivaroxaban [6]. When compared with warfarin, dabigatran and rivaroxaban incurred an increased risk, but only the risk with rivaroxaban reached statistical significance (HR 1.35 [95\% CI 1.23-1.48]) [19]. No statistically significant difference in risk was found when rivaroxaban and dabigatran were compared with each other [6].

\subsection{Intracranial Bleeding Risk}

The risk of intracranial bleeding reduced for all three DOACs compared with warfarin, shown in Tables 3, 5, and 7 [19]. None of the included studies compared intracranial bleeding risk between DOACs or specific intracranial bleeding outcomes. When compared with warfarin, dabigatran displayed the largest reduction in intracranial bleeding risk, with a 53\% reduction reported in one study (HR 0.54 [95\% CI 0.35-0.82]) [19]. Apixaban displayed a 62\% (HR 0.38 [95\% CI 0.25-0.56]) and 36\% (HR 0.64 [95\% CI 0.50-0.80]) intracranial bleeding risk reduction in two studies $[19,22]$.

\subsection{Major Bleeding Risk in Patients Aged > 80 Years}

It is important to understand the impact of DOACs on patients aged $>80$ years, because these patients are more likely to need close monitoring because of their increased fall risk and to require dose adjustment because of renal failure. Only one study reported data specific to patients aged $>80$ years (Table 9) [18]. All DOACs were compared with warfarin; while apixaban was the only DOAC with a major bleeding risk reduction (15\%), it did not reach statistical significance. The analysis of rivaroxaban did show
Table 7 Rivaroxaban bleeding risk

\begin{tabular}{llll}
\hline Rivaroxaban vs. & Major bleed & Gastrointestinal bleed & Intracranial bleed \\
\hline Warfarin & $1.17^{*}(1.10-1.26)[19]$ & $1.35^{*}(1.23-1.48)[19]$ & $0.71^{*}(0.59-0.87)[19]$ \\
& $0.69^{*}(0.51-0.93)[21]$ & & \\
& $0.98(0.83-1.17)[20]$ & & \\
& $1.19(0.99-1.43)[18]$ & & NA \\
Apixaban & $1.82^{*}(1.36-2.43)[20]$ & NA & NA \\
Dabigatran & $1.05(0.74-1.49)[20]$ & $1.20(1.00-1.45)[6]$ & \\
\hline
\end{tabular}

Data are presented as hazard ratio ( $95 \%$ confidence interval)

$N A$ data not available

$* p<0.05$

Table 8 Rivaroxaban bleeding risk forest plot

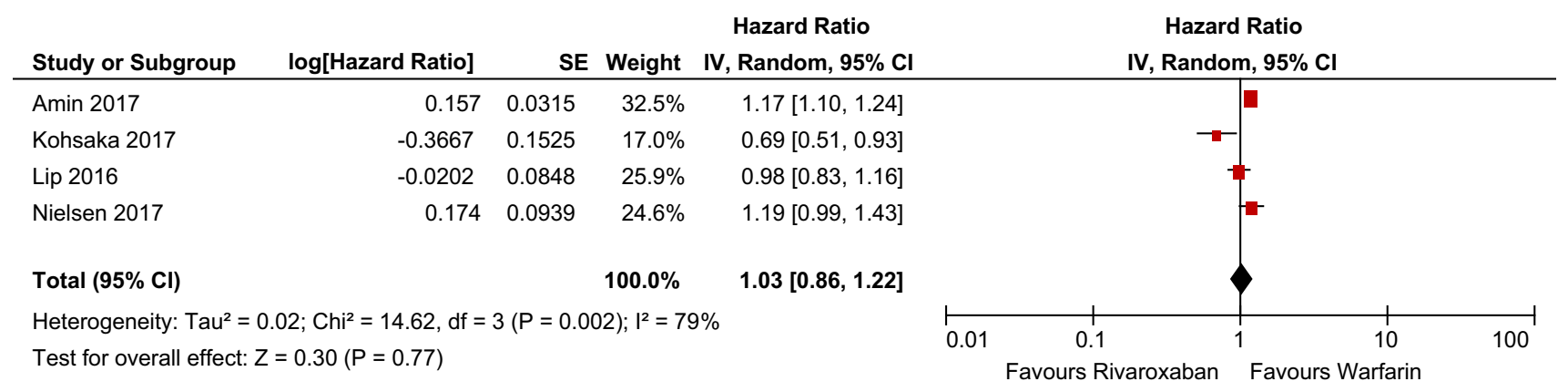

$C I$ confidence interval, $S E$ standard error 


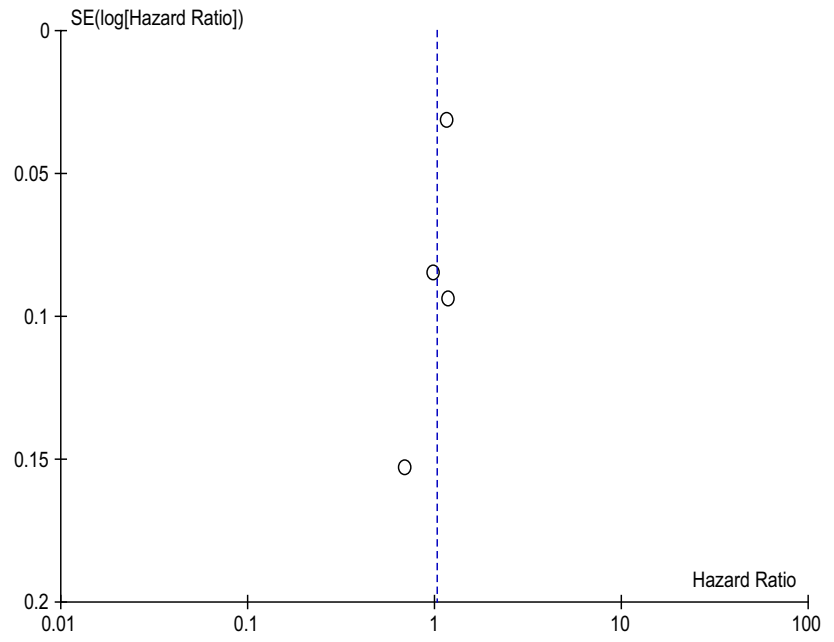

Fig. 4 Rivaroxaban Funnel Plot

statistical significance, with a reported $28 \%$ increased risk of major bleed in patients aged $>80$ years (HR 1.28 [95\% CI 1.00-1.63]) [18]. This study also reported 2.5 years of follow-up and included almost 20,000 individuals aged $>80$ years, all of whom were prescribed a "low-dose" DOAC. This represents a robust study from which strong conclusions regarding safety can be drawn.

\subsection{Cost for Drugs and Reversal Agents}

Costs for a 28-day supply of a DOAC, and the costs for reversal agents, are higher than those for warfarin
(Table 10). The total monthly cost is \$US448.56-469.28 for all DOACs [23]. Warfarin is significantly less expensive, with a range of \$US17.08-27.16 per 28 days of medication, excluding INR testing and other associated expenses [23]. The reversal agent, andexanet alfa, is newer to the market and approved for reversal of apixaban and rivaroxaban at a cost of $\$$ US3300 for every $100 \mathrm{mg}[15$, 23]. The price of andexanet alfa greatly contrasts with that for idariucizumab, the reversal agent for dabigatran, which costs \$US42.00 for every $2.5 \mathrm{~g} / 50 \mathrm{~mL}$ vial. Total dosing for reversal agents often depends upon variables such as dosing of the anticoagulant or INR when a patient is receiving warfarin. This often requires adjustment of the total reversal agent dose and thus can alter the overall cost.

\section{Discussion}

\subsection{Summary of Main Findings}

Apixaban, dabigatran, and rivaroxaban have already been shown to be noninferior to warfarin when considering thromboembolic event risk [3-5]. Bleeding risk is an important consideration when prescribing anticoagulants to patients because the incidence of NVAF increases with advanced age. Beyond the standard bleeding risk (gastrointestinal, intracranial, etc.) associated with increased age, older patients are also at a greater risk for falls and therefore traumatic bleeding events [24]. Current studies recommend that providers continue to prescribe anticoagulants to patients with NVAF despite their fall risk [24, 25]. While DOACs are still recommended in the elderly,
Table 9 Direct oral anticoagulant major bleeding risk compared with warfarin by age

\begin{tabular}{llll}
\hline & Apixaban & Dabigatran & Rivaroxaban \\
\hline$>80$ years & $0.85(0.67-1.08)[18]$ & $1.01(0.84-1.20)[18]$ & $1.28(1.00-1.63)[18]$ \\
\hline
\end{tabular}

Data are presented as hazard ratio (95\% confidence interval)

$N A$ data not available

$* p<0.05$

Table 10 Direct oral anticoagulant and reversal agent standard dosing and cost

\begin{tabular}{llllll}
\hline Drug & Dose & Cost per 28 days & Reversal agent & Total dose & Reversal agent cost \\
\hline Apixaban & $5 \mathrm{mg}$ BID & 469.28 & Andexanet alfa & $880-1760 \mathrm{mg} \mathrm{IV}^{\mathrm{a}}$ & $3300.00 \mathrm{per} 100 \mathrm{mg}$ \\
Dabigatran & $150 \mathrm{mg}$ BID & 448.56 & Idarucizumab & $5 \mathrm{~g} \mathrm{IV}$ & $42.00 \mathrm{per} 2.5 \mathrm{~g} / 50 \mathrm{~mL}$ \\
Rivaroxaban & $20 \mathrm{mg} \mathrm{OD}$ & 469.28 & Andexanet alfa & $880-1760 \mathrm{mg} \mathrm{IV} \mathrm{a}^{\mathrm{a}}$ & $3300.00 \mathrm{per} 100 \mathrm{mg}$ \\
Warfarin & $2-10 \mathrm{mg}^{\mathrm{b}}$ OD & $17.08-27.16$ & Vitamin K and PCC & $2.5-10 \mathrm{mg}^{\mathrm{b}}$ and $50 \mathrm{U} / \mathrm{kg}$ & 66.99 per $5 \mathrm{mg}$ and 2.77 per unit \\
\hline
\end{tabular}

All pricing data are from uptodate.com and presented in \$US [23]

$B I D$ twice daily, INR international normalized ratio, $I V$ intravenous, $O D$ once daily, $P C C$ prothrombin complex concentrate

${ }^{a}$ Dosing dependent upon last apixaban or rivaroxaban dose amount

${ }^{\mathrm{b}}$ Dosing dependent upon INR 
it is important to recognize the exclusion of severe renal failure from this review and analysis. Current strategies in the elderly population overall require an individualized approach that considers all comorbidities before DOAC selection and dose adjustment where necessary [26].

Meta-analysis indicates that apixaban and dabigatran have a significantly lower major bleeding risk, 40 and $27 \%$, respectively, than warfarin. Thus, in terms of major bleeding events, apixaban and dabigatran appear to possess a better safety profile for patients with NVAF aged $>65$ years than warfarin [6, 18-22]. However, meta-analysis indicates that rivaroxaban has comparable major bleeding risk, and some studies reported an increased risk of major bleeding compared with warfarin [18-20]. When directly comparing DOACs, the included studies indicated that apixaban had the lowest risk of major bleeding, followed by dabigatran, and finally rivaroxaban and warfarin [20].

Gastrointestinal and intracranial bleeds are also more likely among the elderly. Two studies reported that apixaban also had the lowest risk of gastrointestinal bleed compared with warfarin, dabigatran, and rivaroxaban $[6,19]$. Dabigatran posed the lowest risk of intracranial bleed [19]. Apixaban and dabigatran both had overall significant reductions in both gastrointestinal and intracranial bleeds, whereas rivaroxaban did not $[6,19,22]$. Only one study reported data for patients aged $<80$ years, and none of the DOACs showed significant bleeding risk reduction or an increase compared with warfarin [18].

The evidence provided by the included studies is strengthened by their robust sample size, reliable data sources, and propensity score-matched cohorts. All data sources were based on insurance claims collected and compiled by either federal or commercial insurance or medical entities. Propensity score-matched cohorts matched patients by their socioeconomic, demographic, and clinical characteristics to best compare the most similar patients. This method also helps reduce the impact of confounders on the outcome, in this case bleeding risk.

\subsection{Strengths and Limitations}

All of the included studies were retrospective in design and thus are limited to the strength of record keeping and data collection. Also common with retrospective studies evaluating medications, physician prescribing methods are done in a nonrandomized manner. In these studies, medication compliance cannot be directly assessed, only implied based on claims data and pharmacy records. In addition, none of the included studies accounted for warfarin dose adjustment and over-the-counter aspirin use, but both could contribute to overall bleeding risk. Bleeding could have also occurred beyond the follow-up periods of the included studies, so conclusions cannot be drawn about the longer-term bleeding risk.
All six studies are considered observational cohorts and thus only associations are assessed and causal relationships cannot be inferred. All six studies use propensity scorematched cohorts, so while confounders may still exist, their impact is minimized.

One study strictly used Medicare data, so these findings may or may not be generalizable to other elderly populations, such as those with commercial or veteran benefits, as they may represent different socioeconomic populations with different comorbidities [19]. To alleviate this, this review also included a study using a database from a US commercially insured older population [20]. It is also important to note that where ICD-9 codes are used to identify the NVAF population in databases, differentiation between permanent, persistent, and paroxysmal AF cannot be made. These specific subsets of NVAF may have different bleeding risks that cannot be assessed using this method.

Finally, the external validity of the included studies is strong, as the setting, study characteristics, and design were all relevant and appropriate. All studies possessed a very robust sample size and used large, reliable databases from which conclusions can be drawn. Further, the risk of bias was assessed using the Cochrane Tool to Assess Risk of Bias in Cohort Studies and all studies had a low risk of bias.

\subsection{Review Limitations and Weaknesses}

The outcomes of this review may be limited by the number of databases searched and the possibility of incomplete retrieval of relevant literature. Because of this, gray literature searches were also performed. Further, this review only included propensity score-matched studies in an effort to ensure a low risk of bias in reporting, but the methods employed may also limit the generalizability of the findings. This study design was selected because of the reproducibility and the low number of randomized controlled trials during preliminary searches. The exclusion of patients with severe renal failure also does not allow for generalization of study conclusions to this population commonly affected by NVAF.

This review is strengthened by the meta-analysis performed and findings that are consistent with those of the review. Further, funnel plots of each DOAC compared with warfarin display heterogeneity and low publication bias influencing the outcome. Two reviewers also provided independent review to minimize the selection bias of included studies.

\section{Conclusion}

This review supports the use of apixaban or dabigatran over rivaroxaban or warfarin because of their lower bleeding risk, as evidenced by a literature review and meta-analysis. 
Providers must undertake significant consideration when prescribing DOACs in order to reduce the risk of thromboembolism appropriately but also factor in the bleeding risk, as it can be equally fatal. It is imperative to have conversations with patients regarding anticoagulant options and risks, because while warfarin has lower direct costs (excluding INR monitoring and associated costs), it may pose increased bleeding risk and requires regular dosing adjustment based upon INR results. The DOACs in patients with normal renal function require less frequent monitoring and have a more manageable dosing schedule, but their cost may be significantly higher for the patient dependent upon insurance coverage. Adding to the considerable cost, reversal agents for apixaban and rivaroxaban are extremely expensive compared with idarucizumab for dabigatran or vitamin $\mathrm{K}$ and prothrombin complex concentrate for warfarin [23]. As a team, the provider and patient must weigh the costs, benefits, risks, and lifestyle changes before selecting an anticoagulant to best ensure safety and compliance. The overall burden and cost to a patient may be lower with apixaban or dabigatran given the lower bleeding risk and need for fewer office visits and monitoring compared with warfarin.

Future research should consider a prospective randomized controlled head-to-head trial of DOACs and include the newest to the market, edoxaban. A study should evaluate both efficacy (thromboembolism risk) and safety (bleeding risk) to best determine superiority of a specific DOAC. This future research should also better stratify demographics, such as age and ethnicity, as well as comorbidities (including but not limited to renal failure, dementia, other hypercoagulable diseases) to enhance the generalizability of results to more specific populations. Additional studies comparing the costs associated with each DOAC and warfarin, including prescriptions, monitoring, and hospitalizations are also needed to assess the potential fiscal burden on the patient and the healthcare system.

\section{Compliance with Ethical Standards}

Conflict of interest JL-F, SB, and EN have no conflicts of interest that are directly relevant to the content of this article.

Funding No sources of funding were used to conduct this study or prepare this manuscript.

Open Access This article is distributed under the terms of the Creative Commons Attribution-NonCommercial 4.0 International License (http://creativecommons.org/licenses/by-nc/4.0/), which permits any noncommercial use, distribution, and reproduction in any medium, provided you give appropriate credit to the original author(s) and the source, provide a link to the Creative Commons license, and indicate if changes were made.

\section{References}

1. January C, Wann L, Alpert J, Calkins H, Cigarroa J, Cleveland JJ, et al. AHA/ACC/HRS guideline for the management of patients with atrial fibrillation: executive summary: a report of the American College of Cardiology/American Heart Association Task Force on Practice Guidelines and the Heart Rhythm Society. Circulation. 2014;130:2071-104.

2. Giugliano RP, Ruff CT, Braunwald E, Murphy SA, Wiviott SD, Halperin JL, et al. Edoxaban versus warfarin in patients with atrial fibrillation. N Engl J Med. 2013;369:2093-104.

3. Patel M, Mahaffey K, Garg J, Pan G, Singer D, Hacke W, et al. Rivaroxaban versus warfarin in nonvalvular atrial fibrillation. NEJM. 2011;365:883-91.

4. Granger C, Alexander J, McMurray J, Lopes R, Hylek E, Hanna $\mathrm{M}$, et al. Apixaban versus warfarin in patients with atrial fibrillation. NEJM. 2011;365:981-92.

5. Connolly S, Ezekowitz M, Yusuf S, Eikelboom J, Oldgren J, Parekh A, et al. Dabigatran versus warfarin in patients with atrial fibrillation. NEJM. 2009;361:1139-51.

6. Neena A, Noseworthy P, Yao X, Sangaralingham L, Shah N. Gastrointestinal safety of direct oral anticoagulants: a large population-based study. Gastroenterology. 2017;152:1014-22.

7. American Heart Association Statistics Committee and Stroke Statistics Subcommittee. Heart disease and stroke statistics-2015 update: a report from the American Heart Association. Circulation. 2015;131:e29-322.

8. Lip G, Pan X, Kamble S, Kawabata H, Mardekian J, Masseria C, et al. Major bleeding risk among non-valvular atrial fibrillation patients initiated on apixaban, dabigatran, rivaroxaban or warfarin: a "real-world" observational study in the United States. Int J Clin Pract. 2016;70:752-63.

9. Which oral anticoagulant for atrial fibrillation. Med Lett Drugs Ther. 2016;58(1492):45-6.

10. Winkelmayer WC, Patrick AR, Liu J, Brookhart MA, Setoguchi $\mathrm{S}$. The increasing prevalence of atrial fibrillation among hemodialysis patients. J Am Soc Nephrol. 2011;22:349-57.

11. Renoux C, Patenaude V, Suissa S. Incidence, mortality, and sex differences of non-valvular atrial fibrillation: a population-based study. J Am Heart Assoc. 2014;3:1-8.

12. World Health Organization Department of Ageing and Life. WHO global report on falls prevention in older age. Geneva: Switzerland; 2008.

13. Suarez Fernandez C, Formiga F, Camafort M, Cepeda Rodrigo M, Diez-Manglano J, Pose Reino A, et al. Antithrombotic treatment in elderly patients with atrial fibrillation: a practical approach. BMC Cardiovasc Disord. 2015;15:143. https://doi.org/10.1186/ s12872-015-0137-7

14. American College of Cardiology. Reversing oral anticoagulants: new possibilities. Cardiology Magazine: Lingering Misconceptions; 2017.

15. Siegal DM, Curnutte JT, Connolly SJ, Lu G, Conley PB, Wiens $\mathrm{BL}$, et al. Andexanet alfa for the reversal of factor Xa inhibitor activity. N Engl J Med. 2015;373:2413-24.

16. Pollack CJ, Reilly P, Eikelboom J, Glund S, Verhamme P, Bernstein R, et al. Idarucizumab for dabigatran reversal. N Engl J Med. 2015;373:511-20.

17. Larsen T, Skoth F, Nielsen P, Kjaeldgaard J, Lip G. Comparative effectiveness and safety of non-vitamin $\mathrm{K}$ antagonist oral anticoagulant and warfarin in patients with atrial fibrillation: propensity weighted nationwide cohort study. BMJ. 2016;353:1-9.

18. Nielsen PB, Skjoth F, Sogaard M, Kjaeldgaard JN, Lip GY, Larsen TB. Effectiveness and safety of reduced dose non-vitamin $\mathrm{K}$ antagonist oral anticoagulants and warfarin in patients with atrial 
fibrillation: propensity weighted nationwide cohort study. BMJ. 2017;356:j510. https://doi.org/10.1136/bmj.j510

19. Amin A, Keshishian A, Trocio J, Dina O, Le H, Rosenblatt L, et al. Risk of stroke/systemic embolism, major bleeding and associated costs in non-valvular atrial fibrillation patients who initiated apixaban, dabigatran or rivaroxaban compares with warfarin in the United States Medicare population. Curr Med Res Opin. 2017;33:1595-604.

20. Lip G, Keshishian A, Kamble S, Pan X, Mardekian J, Horblyuk $\mathrm{R}$, et al. Real-world comparison of major bleeding risk among non-valvular atrial fibrillation patients initiated on apixaban, dabigatran, rivaroxaban, or warfarin. Thromb Haemost. 2016;116:975-86.

21. Kohsaka S, Murata T, Izumi N, Katada J, Wang F, Terayama Y. Bleeding risk of apixaban, dabigatran, and low-dose rivaroxaban compared with warfarin in Japanese patients with non-valvular atrial fibrillation: a propensity matched analysis of administrative claims data. Curr Med Res Opin. 2017;33:1955-63.
22. Li X, Deitelzweig S, Keshishian A, Hamilton M, Horblyuk R, Gupta K, et al. Effectiveness and safety of apixaban versus warfarin in non-valvular atrial fibrillation patients in "real-world" clinical practice: a propensity-matched analysis of 76,940 patients. Thromb Haemost. 2017;117:1072-82.

23. Basow D. UpToDate. Waltham: UpToDate; 2018. https://www. uptodate.com/. Accessed 17 May 2019.

24. Sellers MB, Newby LK. Atrial fibrillation, anticoagulation, fall risk, and outcomes in elderly patients. Am Heart J. 2011;161:241-6.

25. Boltz MM, Podany AB, Hollenbeak CS, Armen SB. Injuries and outcomes associated with traumatic falls in the elderly population on oral anticoagulant therapy. Injury. 2015;46:1765-71.

26. Kundu A, Sardar P, Chatterjee S, Aronow WS, Owan T, Ryan JJ. Minimizing the risk of bleeding with NOACs in the elderly. Drugs Aging. 2016;33:491-500. 